\title{
Four new species of the trapdoor spider genus Conothele Thorell, 1878 (Araneae, Halonoproctidae) from China
}

\author{
Hao Liu', Xin $\mathrm{Xu}^{1,2}$, Zengtao Zhang', Fengxing Liu', Daiqin $\mathrm{Li}^{3}$ \\ I State Key Laboratory of Biocatalysis and Enzyme Engineering, Centre for Behavioural Ecology and Evolution \\ (CBEE), School of Life Sciences, Hubei University, 368 Youyi Road, Wuhan 430062, Hubei Province, China \\ 2 College of Life Sciences, Hunan Normal University, 36 Lushan Road, Changsha 410081, Hunan Province, China \\ 3 Department of Biological Sciences, National University of Singapore, 14 Science Drive 4, 117543, Singapore \\ Corresponding author: Xin Xu (xuxin_09@163.com); Daiqin Li (dbslidq@nus.edu.sg)
}

Academic editor: C. Hamilton | Received 29 December 2018 | Accepted 30 January 2019 | Published 1 April 2019

http://zoobank.org/EDCF57EA-B7D9-4C8F-98F4-6AB6A8F6FDD4

Citation: Liu H, Xu X, Zhang Z, Liu F, Li D (2019) Four new species of the trapdoor spider genus Conothele Thorell, 1878 (Araneae, Halonoproctidae) from China. ZooKeys 833: 133-150. https://doi.org/10.3897/zookeys.833.32736

\begin{abstract}
Herein four species of the trapdoor spider genus Conothele Thorell, 1878 collected from China are described as new to science based on the female genital morphology: C. baisha sp. n. (Hainan Province), C. baoting sp. n. (Hainan Province), C. linzhi sp. n. (Tibet), and C. jinggangshan sp. n. (Jiangxi Province). For two Hainan species, C. baisha sp. n. and C. baoting sp. n., between which it is difficult to distinguish solely based on female genital morphology, additional diagnoses derived from species-specific nucleotide substitution information and genetic distances using the mitochondrial gene, cytochrome $\mathrm{c}$ oxidase subunit I are provided.
\end{abstract}

\section{Keywords}

Araneae, China, COI, DNA barcode, Mygalomorphae, taxonomy

\section{Introduction}

Conothele Thorell, 1878 is a genus of trapdoor spiders belonging to the family Halonoproctidae Pocock, 1901 (Opisthothelae: Mygalomorphae) that was recently elevated from the family Ctenizidae based on molecular-based evidence (Godwin et al. 2018). 
Like many poor-dispersal, ground-dwelling trapdoor spiders (although some species of Ummidia Thorell, 1875 disperse by ballooning (Coyle 1985; Fisher et al. 2014)), Conothele spiders construct underground burrows which are lined with silk and opened to the surface with a trapdoor. The trapdoor is usually covered with a layer of soil, leaf litter, and/or moss, which blend well in the surrounding environment, making them difficult to locate in nature (Fig. 1; Bond and Coyle 1995; Xu et al. 2017a; Yang and Xu 2018).

Conothele was previously placed in the family Ctenizidae. However, the two Ctenizidae subfamilies, Ctenizinae and Ummidiinae (Raven 1985; Ortiz 2007), and even the entire family were not monophyletic (Hedin and Bond 2006; Ayoub et al. 2007; Bond et al. 2012; Opatova et al. 2013). Recently, Godwin et al. (2018) relimited the whole family and subfamilies based on molecular phylogenetic evidence, and split it into two families, Halonoproctidae and Ctenizidae. Halonoproctidae now comprises six genera and 87 species belonging to two subfamilies, Ummidiinae Ortiz, 2007 (Conothele, Latouchia Pocock, 1901, and Ummidia) and Halonoproctinae Pocock 1901 (Bothriocyrtum Simon, 1891, Cyclocosmia Ausserer, 1871, and Hebestatis Simon, 1903) (Godwin et al. 2018; World Spider Catalog 2019).

The two ummidiin genera Conothele and Ummidia share some common morphological and behavioral characters, thus they were considered as undistinguishable (Main 1985; Decae 2010). One of the most obvious shared features by two genera is the presence of a saddle depression on tibia III (Gertsch 1979; Coyle 1981; Ortiz 2007), leading some authors to consider both genera as synonyms (Decae 2010). However, these two genera are completely separated geographically, with Conothele being distributed in the Orient and Australasian regions, and Ummidia being found in the New World and Mediterranean regions (Xu et al. 2017a; Godwin et al. 2018; Yang and Xu 2018). In addition, they are reciprocally monophyletic, and currently considered as valid genera based on phylogenetic analyses (Godwin et al. 2018).

Conothele contains 26 described species that are widely distributed in the Orient (China, India, Japan, Laos, Myanmar, Sumatra) and Australasia (World Spider Catalog 2019). Until now, only seven species have been described from China primarily based on either female or male morphology (World Spider Catalog 2019), including C. taiwanensis (Tso, Haupt \& Zhu, 2003) (ð̊+; Taiwan Province), C. baiyunensis (Xu, Xu \& Liu, 2017) (†; Guangzhou Province), C. daxinensis (Xu, Xu \& Li, 2017) (क; Guangxi Province), C. sidiechongensis (Xu, Xu \& Liu, 2017) (㕜; Yunnan Province), C. yunding-

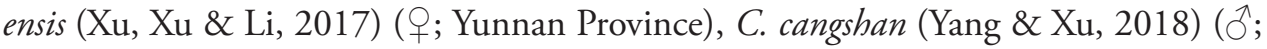

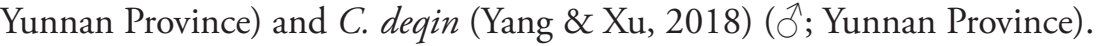

In this study, we diagnosed and described four new Conothele species collected in China based on female morphology as we were unable to obtain adult males (Fig. 2). As in other halonoproctid studies (Xu et al. 2017a; Yang and Xu 2018), both male and female morphology should be described for a new species; however, often it is impractical or impossible to collect adult males by direct searching or by excavating burrows. The standard DNA alignment of the mitochondrial cytochrome $c$ oxidase subunit (COI), which provides the species-specific nucleotide substitution information in the animal barcoding gene region, has been widely used to diagnose species 

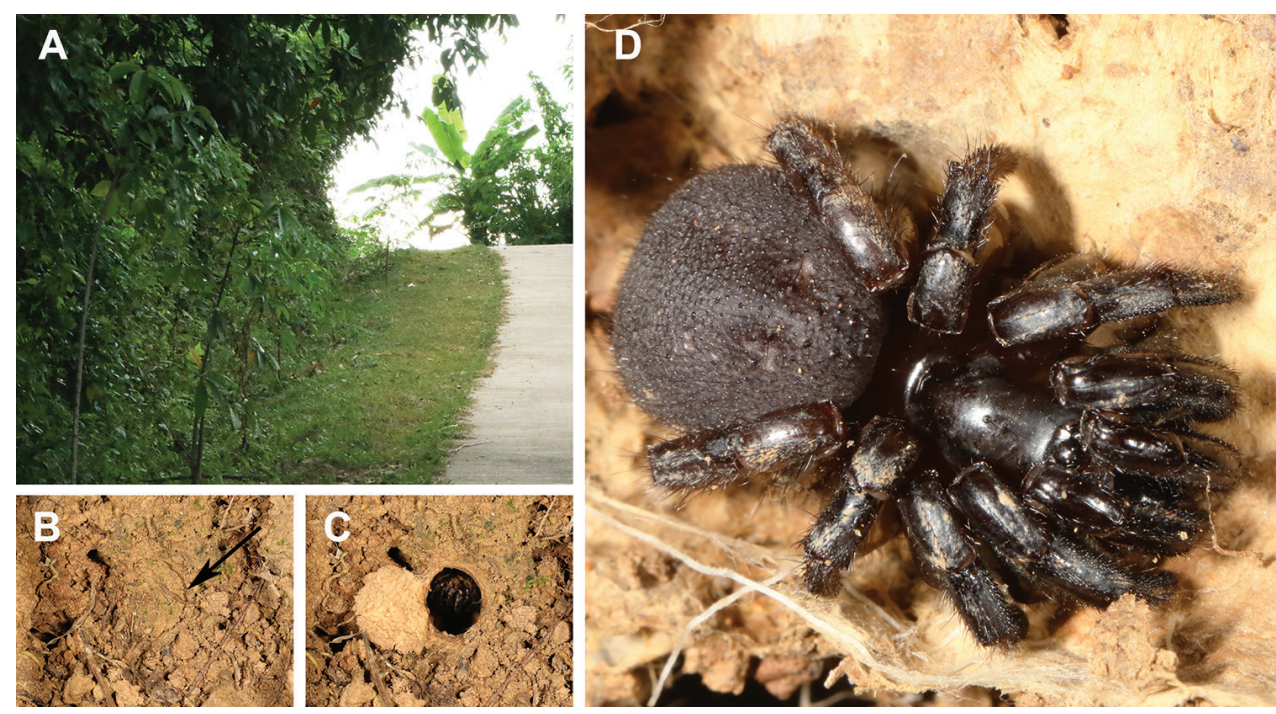

Figure I. Microhabitat, burrow with a trapdoor, and general somatic morphology of Conothele baisha sp. n. A microhabitat $\mathbf{B}, \mathbf{C}$ burrow exterior $\mathbf{B}$ the trapdoor with the door closed $\mathbf{C}$ the trapdoor with door open D female (LH-2017-089; Jishi Village, Changjiang County, Hainan Province, China).

(Brower 2010; Cook et al. 2010; Planas and Ribera 2015; Xu et al. 2015, 2017b). Therefore, for the two new species from Hainan Province (Conothele baisha sp. n. and C. baoting sp. n.) that show similar morphology and considerable intraspecific variations in female genitalia, we provided additional evidence of species-specific nucleotide substitutions and genetic distances based on COI to support our identifications and for future verification of males.

\section{Materials and methods}

All specimens were collected from Tibet, Hainan, Jiangxi Provinces, China (Fig. 2). The right four legs of adult females were removed and stored in $100 \%$ ethanol at $-80{ }^{\circ} \mathrm{C}$ for the molecular work. The rest of each specimen was stored as a voucher in $75-80 \%$ ethanol for morphological examination. All the voucher specimens were examined under an Olympus SZX16 stereomicroscope, and they were photographed using a Leica M205C digital microscope. Genitalia were cleaned by Protease K digest for $3 \mathrm{hrs}$ at $56{ }^{\circ} \mathrm{C}$. All the voucher specimens were deposited at the CBEE (Centre for Behavioural Ecology and Evolution), School of Life Sciences, Hubei University, Wuhan, China. All measurements were carried out under a Leica M205C digital microscope and given in millimeters. Standard measurements were made following Decae (2010). Measurements of legs and palps are given in the following order: Leg total length (femur + patella + tibia + metatarsus + tarsus), palp total length (femur + patella + tibia + tarsus). 


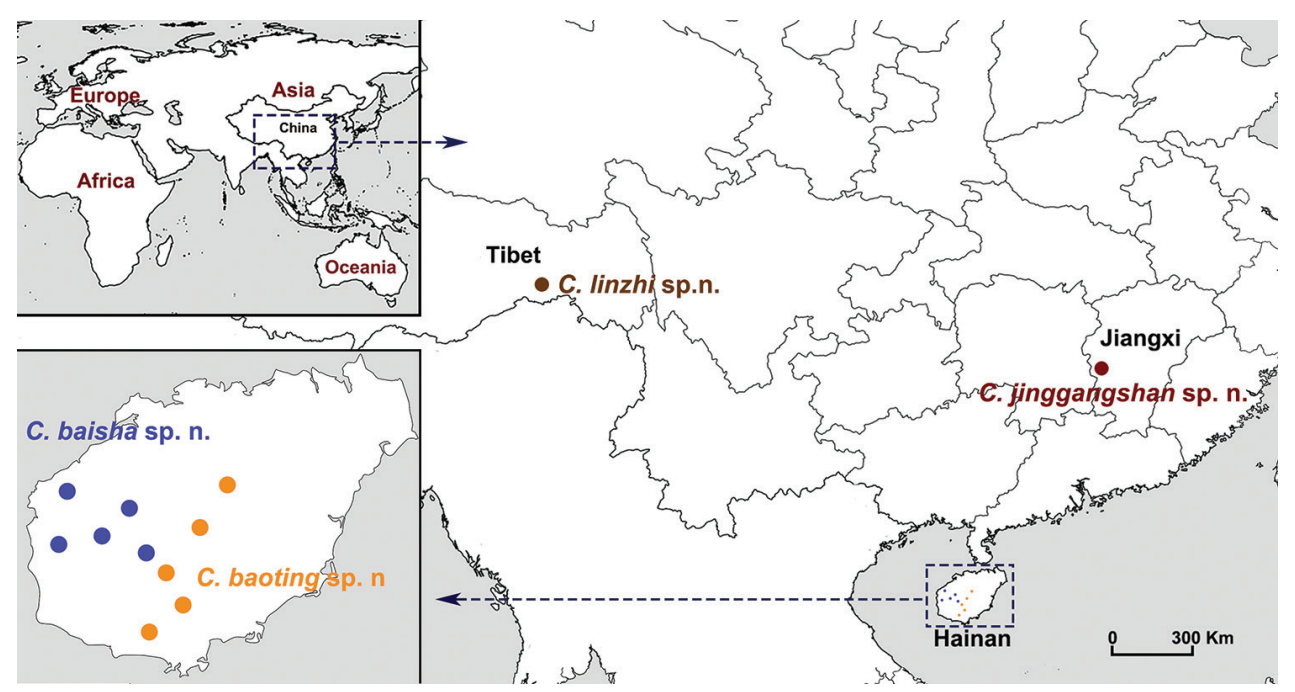

Figure 2. A map showing the distribution of four new species.

Abbreviations used are:

$\begin{array}{llll}\text { ALE } & \text { anterior lateral eye; } & \text { PMS } & \text { posterior median spinneret; } \\ \text { AME } & \text { anterior median eye; } & \text { PLS } & \text { posterior lateral spinneret; } \\ \text { PLE } & \text { posterior lateral eye; } & \text { TL } & \text { total length (including cheli- } \\ \text { PME } & \text { posterior median eye; } & & \text { cerae but excluding spinnerets). } \\ \text { MOA } & \text { median ocular area; } & & \end{array}$

We extracted the total genomic DNA using the universal genomic DNA extraction kit (CWBIO) from one or two right legs per specimen depending on the size of the legs. The $25 \mu \mathrm{L}$ PCR reaction included $12.5 \mu \mathrm{l} 2 \times$ TaqMaster Mix (TIANGEN), $9.5 \mu$ d double-distilled $\mathrm{H}_{2} \mathrm{O}\left(\mathrm{ddH}_{2} \mathrm{O}\right), 1 \mu \mathrm{l}$ genomic DNA and $1 \mu \mathrm{l}$ of each forward and reverse primer $(10 \mu \mathrm{M})$. The primer pairs of COI were LCO1490 (5'-GGTCAACAAATCATAAAGATATTGG-3') and HCO2198 (5'-TAAACTTCAGG GTGACCAAA AAATCA-3') (Folmer et al. 1994). The PCR reaction protocol: initial denaturation at $94{ }^{\circ} \mathrm{C}$ for $5 \mathrm{~min}$; 35 cycles of denaturation at $94{ }^{\circ} \mathrm{C}$ for $30 \mathrm{~s}$, annealing at $40{ }^{\circ} \mathrm{C}$ for $45 \mathrm{~s}$ and elongation at $72{ }^{\circ} \mathrm{C}$ for $1 \mathrm{~min}$, and final extension at $72{ }^{\circ} \mathrm{C}$ for $10 \mathrm{~min}$. The PCR products were visualized by agarose gel electrophoresis (1\% agarose). All PCR products were purified and sequenced at the TSINGKE Biological Technology (Wuhan China) or Sunny Biological (Shanghai China). The species-specific nucleotide substitutions in the standard DNA barcode alignment and genetic distances were identified using MEGA v6 (Tamura et al. 2013; Xu et al. 2017b). 


\section{Taxonomy}

\section{Genus Conothele Thorell, 1878}

Type. Conothele malayana (Doleschall 1859): 5, pl. 5, fig. 8 (described female).

Diagnosis. The genus Conothele can be distinguished from all other Halonoproctidae genera other than Ummidia by the presence of a saddle depression on tibia III (Coyle 1981; Ortiz 2007; Decae 2010). Conothele differs from Ummidia by their burrowing habits. The former constructs a short, parallel to the surface of ground, superficial burrow, whereas the latter digs a several centimeters long burrow in the soil (Haupt 2006). Moreover, the geographical ranges of Ummidia and Conothele are completely separated, with Conothele being distributed in the Orient and Australasian regions, and with Ummidia being distributed in the New World and Mediterranean regions (Xu et al. 2017a; Godwin et al. 2018; Yang and Xu 2018; World Spider Catalog 2019).

\section{Conothele linzhi sp. $\mathrm{n}$.}

http://zoobank.org/93117D0B-1A52-4CC3-9E67-044B44BB7DAF

Fig. 3

Holotype. Female (LH-2017-051), collected in Baishuwang Garden Roadside, Bayi Town, Linzhi City, Tibet, China, 29.6106N, 94.4040E, 2980 m a.s.l., 14 July 2017, collected by FX Liu, ZT Zhang, J Chen and J Liu (CBEE).

Paratypes. 3 females (LH-2017-046, LH-2017-048, LH-2017-050), collected at the same locality as the holotype (CBEE).

Diagnosis. Females of C. linzhi sp. n. can be distinguished from those of the other Conothele species by an obviously large irregularly duct-like sigillum in the sternum center (Fig. 3C); by the terminal lobes of spermathecae hemisphere-shaped; by the distal part of stalks Z-shaped and tilted slightly anteriorly (Fig. 3G-J).

Description. TL 19.26; chelicerae length 2.51, carapace 7.39 long, 7.15 wide; opisthosoma 9.43 long, 7.38 wide. Carapace brownish black, glabrous, with a few slender setae on or behind the eye tubercle. Caput arched. Fovea deep and darker (Fig. $3 \mathrm{~A})$. Eight eyes in two rows, with the anterior eye row procurved, and the posterior eye row straight (Fig. 3E); eye group 0.93 long, 1.48 wide; ALE-AME 0.29, AMEAME 0.18, PLE-PME 0.08, PME-PME 0.38; MOA 0.64 long, front width 0.56, back width 0.82; ALE: AME: PLE: PME (0.48: 0.19: 0.33: 0.21). Many slender setae on clypeus (Fig. 3E). Chelicerae black (dorsal view); inner margin with 6 teeth, outer margin with 9 teeth. Labium, coxae of palp and sternum brownish black (Fig. 3C). Labium 1.27 long, 1.47 wide, with 19 conspicuous cuspules. Coxae of palp 2.78 long, 1.86 wide, with approx. 68 conspicuous cuspules (the right one, ventral view) (Fig. 3C). Sternum 4.60 long, 4.65 wide, with an obviously large, irregularly shaped sigillum in the center and with many setae (Fig. 3C). 

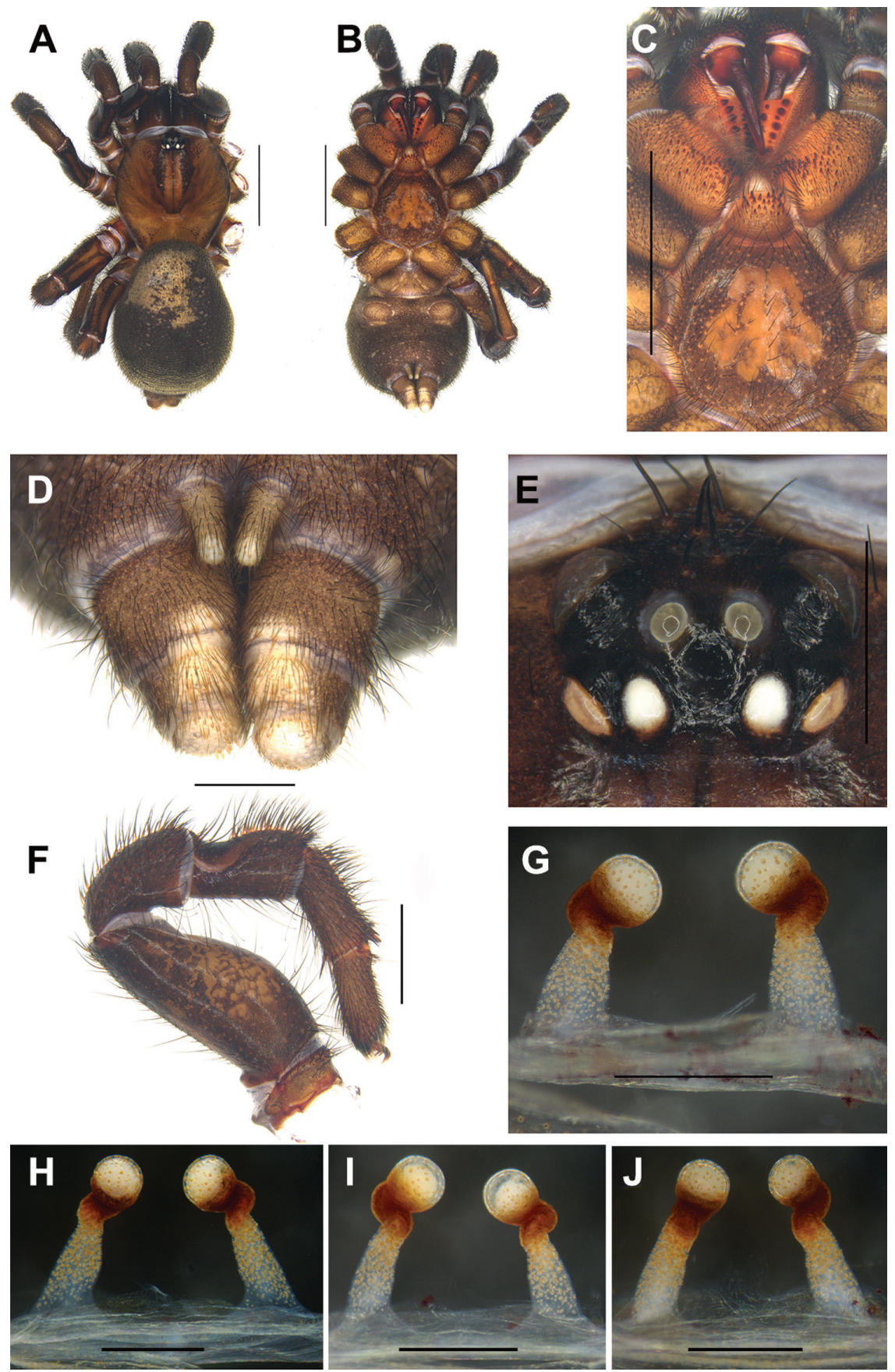

Figure 3. General somatic morphology and female genitalia of Conothele linzhi sp. n. A-G holotype (LH-2017-051) A dorsal view B ventral view C chelicerae, labium, coxae of palp and sternum, ventral view $\mathbf{D}$ spinnerets, ventral view $\mathbf{E}$ eyes, dorsal view $\mathbf{F}$ left leg III, prolateral view $\mathbf{G}-\mathbf{J}$ female genitalia, dorsal view H-J paratypes $\mathbf{H}$ (LH-2017-046) I (LH-2017-048) J (LH-2017-050). Scale bars: 5 mm (A-C); $1 \mathrm{~mm}(\mathbf{D}, \mathbf{E}) ; 2 \mathrm{~mm}(\mathbf{F}) ; 0.5 \mathrm{~mm}(\mathbf{G}-\mathbf{J})$. 
Legs brownish black, with long and short black dense setae. Tibia III with a saddlelike depression dorsally on the basal part, and the depression is smaller than that of the other Conothele species (Fig. 3F). Palp with a single tarsal claw and a denticle on the claw. Legs each with three tarsal claws, paired claws with one denticle. Leg formula: IV, I, II, III. Measurements of palp and legs: palp $13.25(4.99+2.02+3.51+2.73)$, leg I $15.25(5.65+2.66+3.61+2.12+1.21)$, leg II $12.74(4.52+2.13+2.65+2.06+1.38)$, leg III $12.70(4.52+1.84+2.33+2.00+2.01)$, leg IV $16.22(5.22+2.24+2.77+3.13+2.86)$.

Opisthosoma ellipsoid and black, scattered with thick and slender black setae. Spinnerets brown (Fig. 3D), PMS one-segmented, 0.86 long, PMS-PMS 0.23; PLS divided into three sections, 2.17 long. Female genitalia with a pair of spermathecae slightly tilted to the middle; the terminal lobes of spermathecae hemisphere-shaped; stalks sclerotized and Z-shaped distally, and tilted slightly anteriorly (Fig. 3G-J).

Male. Unknown.

Etymology. The species epithet, a noun in apposition, refers to the type locality.

Distribution. Tibet (Linzhi City).

\section{Conothele jinggangshan sp. $\mathrm{n}$.}

http://zoobank.org/B259FF23-9B58-40C0-8F4B-11905347CDA9

Fig. 4

Holotype. Female (LH-2017-225), collected in Revolutionary Martyrs Cemetery, Ciping Town, Jinggangshan City, Jian City, Jiangxi Province, China, 26.5881N, 114.1599E, 910 m a.s.l., 12 September 2017, collected by FX Liu, F Li (CBEE).

Diagnosis. Female of $C$. jinggangshan sp. $n$. can be distinguished from those of the other Conothele species by the sternum with a pair of obvious elliptic sigilla (Fig. 4C); by the distal part of stalks which are outwardly and then inwardly bend, somewhat semi-circle-like (Fig. 4G).

Description. TL 13.74; chelicerae length 1.77, carapace 6.62 long, 5.40 wide; opisthosoma 6.89 long, 5.70 wide. Carapace dark brown, glabrous, with a few slender setae on or behind the eye tubercle (Fig. 4A). Caput arched. Fovea deep and dark (Fig. $4 \mathrm{~A})$. Eye tubercle black. Eight eyes in two rows, with the anterior eye row procurved, and the posterior eye row slightly recurved (Fig. 4E); eye group 0.73 long, 1.38 wide; ALE-AME 0.20, AME-AME 0.14, PLE-PME 0.02, PME-PME 0.46; MOA 0.51 long, front width 0.48, back width 0.86; ALE: AME: PLE: PME (0.34: 0.17: 0.30: 0.19). Four slender setae on clypeus (Fig. 4E). Chelicerae dark brown (dorsal view); inner margin with five teeth, outer margin with seven teeth. Labium, coxae of palp and sternum brown (Fig. 4C). Labium 0.82 long, 1.00 wide, with four conspicuous cuspules. Coxae of palp 2.03 long, 1.31 wide, with approx. 41 conspicuous cuspules (the right one, ventral view) (Fig. 4C). Sternum 3.09 long, 2.74 wide, with a pair of obvious elliptic sigilla and with small number of setae (Fig. 4C).

Legs brown, light brown ventrally, with long and short brown sparse setae. Basal part of tibia III with saddle-like depression dorsally (Fig. 4F). Palp with a single tar- 

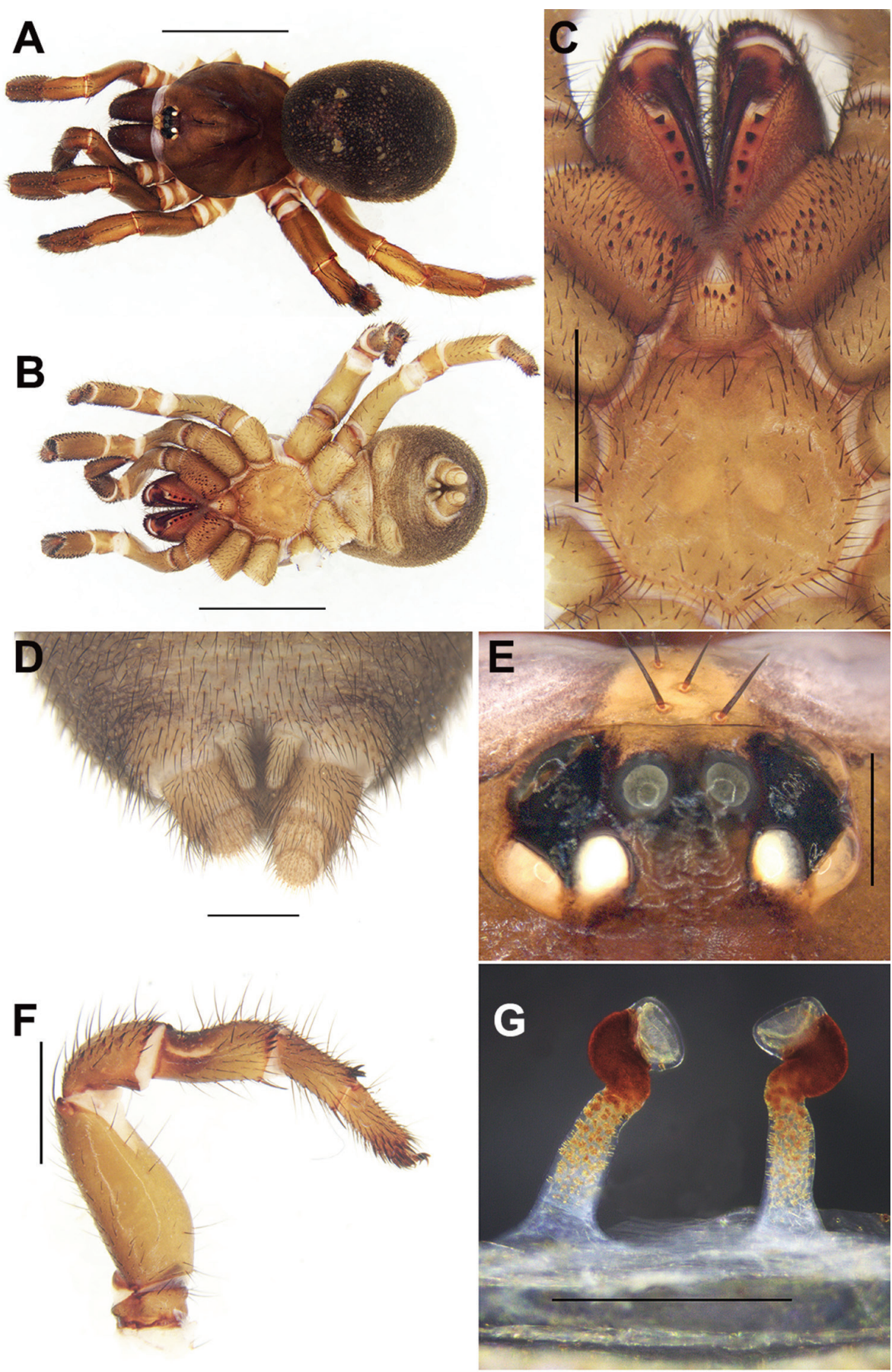

Figure 4. General somatic morphology and female genitalia of Conothele jinggangshan sp. $\mathrm{n}$. (holotype, LH-2017-225) A dorsal view B ventral view C chelicerae, labium, coxae of palp and sternum, ventral view $\mathbf{D}$ spinnerets, ventral view $\mathbf{E}$ eyes, dorsal view $\mathbf{F}$ left leg III, prolateral view $\mathbf{G}$ female genitalia, dorsal view. Scale bars: $5 \mathrm{~mm}(\mathbf{A}, \mathbf{B}) ; 1 \mathrm{~mm}(\mathbf{D}) ; 2 \mathrm{~mm}(\mathbf{C}, \mathbf{F}) ; 0.5 \mathrm{~mm}(\mathbf{E}, \mathbf{G})$. 
sal claw and with two denticles on the claw. Legs each with three tarsal claws, paired claws with one denticle. Leg formula: IV, I, II, III. Measurements of palp and legs: palp $9.07(3.40+1.42+2.27+1.98)$, leg I $9.89(3.65+1.93+2.41+1.04+0.86)$, leg II 9.40 $(3.21+1.66+2.13+1.23+1.17)$, leg III $9.35(3.20+1.29+2.01+1.36+1.49)$, leg IV12.09 $(4.14+1.74+2.50+2.16+1.55)$.

Opisthosoma ellipsoid, black, scattered with slender short black setae. Spinnerets brown (Fig. 4D), PMS short and one-segmented, 0.64 long, PMS-PMS 0.19; PLS divided into three sections, 1.38 long. Genitalia with a pair of spermathecae, each stalk slender, long, distally sclerotized and folded, which is first bent outwards and then inwards, semi-circle-like; with bowl-shaped lobes (Fig. 4G).

Male. Unknown.

Etymology. The species epithet, a noun in apposition, refers to the type locality.

Distribution. Jiangxi Province (Jinggangshan City).

\section{Conothele baisha sp. $\mathrm{n}$.}

http://zoobank.org/464B6E9B-B04A-49F8-8516-AE79A33A12A7

Figs 5, 6

Holotype. Female (LH-2017-136), collected in Nanmeiling, Yacha Town, Baisha County, Hainan Province, China, 19.1075N, 109.4227E, 250 m a.s.l., 10 August 2017, collected by FX Liu, D Li, ZT Zhang, X Xu (CBEE).

Paratypes. 2 females (LH-2017-128, LH-2017-135) collected at the same locality as the holotype (CBEE); 1 female (LH-2017-080), collected in Yalong Village, Tianan Township, Donghe Town, Dongfang City, Hainan Province, China, 18.9947N, 108.8976E, 170 m a.s.l., 5 August 2017; 1 female (LH-2017-089), collected in Jishi Village, Changjiang County, Hainan Province, China, 19.2305N, 109.0730E, 170 m a.s.l., 6 August 2017; 1 female (LH-2017-090), collected in Bawangling National Forest Park, Baoshan village, Changjiang County, Hainan Province, China, 19.0757N, 109.0822E, $210 \mathrm{~m}$ a.s.l., 7 August 2017; 1 female (LH-2017-161), collected in Shiyixinyi Village, Wuzhishan City, Hainan Province, China. 18.9122N, 109.5118E, 290 m a.s.l., 11 August 2017, all collected by FX Liu, D Li, ZT Zhang, X Xu (CBEE).

Diagnosis. Female genitalia of $C$. baisha sp. n. resembles $C$. daxinensis $(\mathrm{Xu}, \mathrm{Xu} \&$ $\mathrm{Li}, 2017)$, but can be distinguished from the latter by the spermathecae with each stalk sturdy, short, simple and direct (Fig. 5G). It can be also distinguished from C. baoting sp. n. by short stalks without the trench between the distal part of the stalks and the lobes. Moreover, C. baisha sp. n. can be distinguished from C. baoting sp. n. by the following unique nucleotide substitutions in the standard DNA barcode alignment: A (13), G (97), A (134), T (157), A (172), G (196), C (205), A (223), T (224), A (253), G (280), C (302), G (304), C (322), A (421), G (424), A (502), G (520), A (592), A (634), G (637).

Description. TL10.35; chelicerae length 1.49 , carapace 4.76 long, 4.28 wide; opisthosoma 4.83 long, 4.22 wide. Carapace brown, glabrous, with a few slender setae on or behind the eye tubercle (Fig. 5A). Caput arched. Fovea deep and brown (Fig. 5A). 

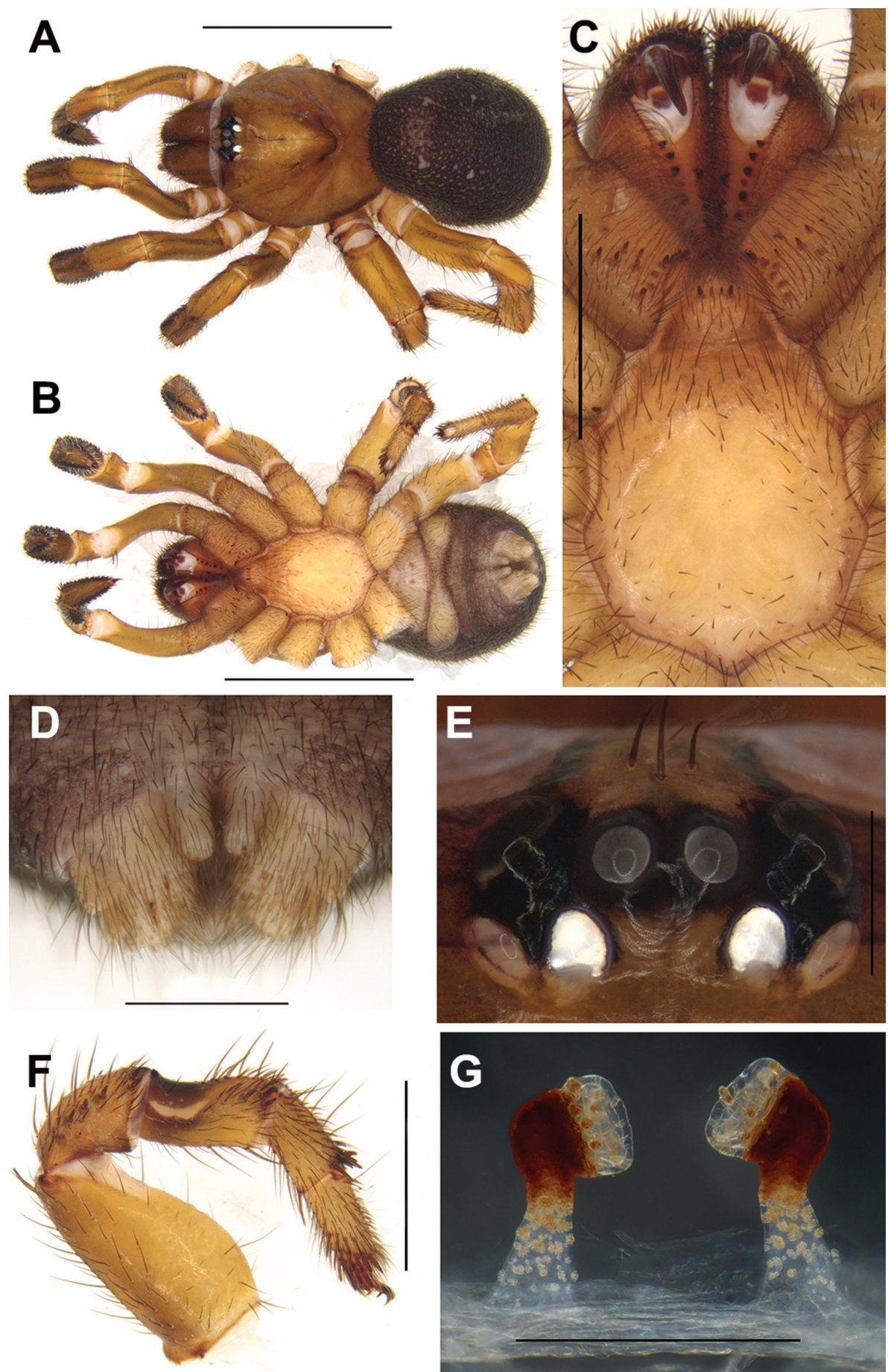

Figure 5. General somatic morphology and female genitalia of Conothele baisha sp. n. holotype (LH2017-136) A dorsal view B ventral view C chelicerae, labium, coxae of palp and sternum, ventral view $\mathbf{D}$ spinnerets, ventral view $\mathbf{E}$ eyes, dorsal view $\mathbf{F}$ left leg III, prolateral view $\mathbf{G}$ female genitalia, dorsal view. Scale bars: $5 \mathrm{~mm}(\mathbf{A}, \mathbf{B}) ; 1 \mathrm{~mm}(\mathbf{D}) ; 2 \mathrm{~mm}(\mathbf{C}, \mathbf{F}) ; 0.5 \mathrm{~mm}(\mathbf{E}, \mathbf{G})$. 

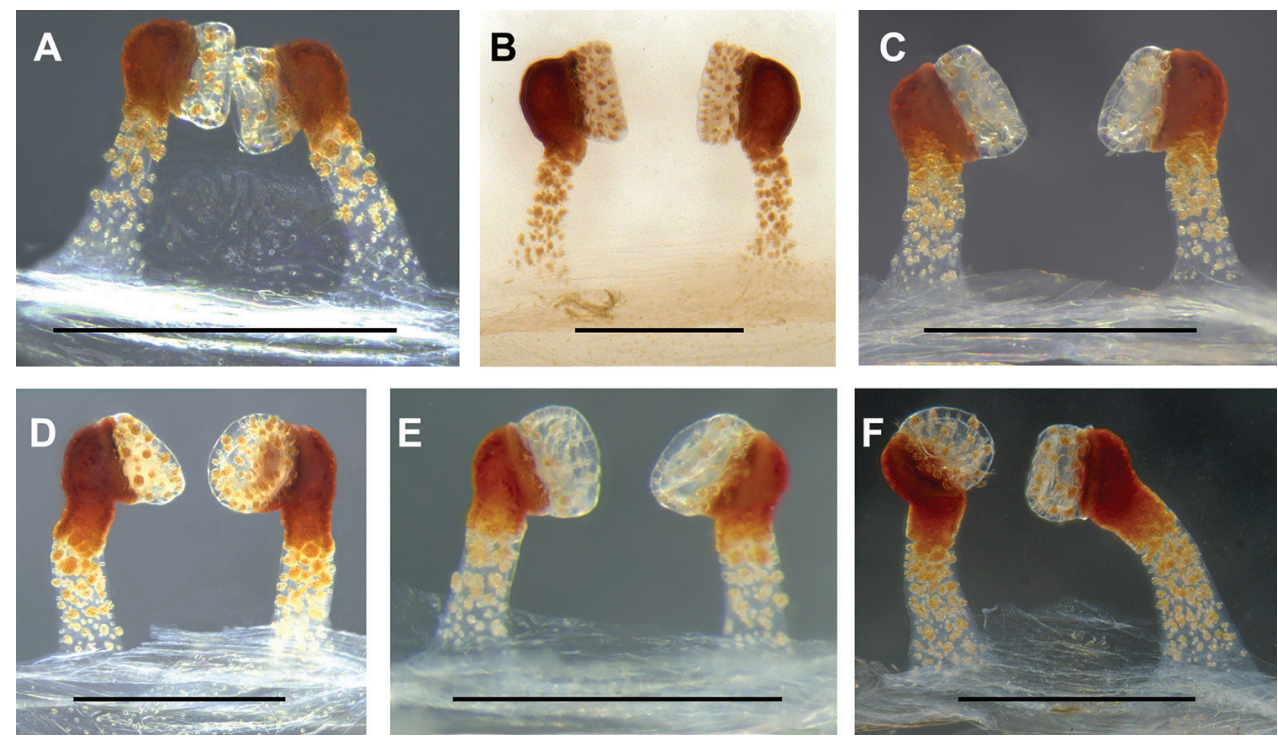

Figure 6. Female genitalia of the paratypes of Conothele baisha sp. n. showing the intraspecific variations in spermathecae. A (LH-2017-080) B (LH-2017-089) C (LH-2017-090) D (LH-2017-128) E (LH2017-135) F (LH-2017-161), dorsal view. Scale bars: $0.5 \mathrm{~mm}$.

Eye tubercle black. Eight eyes in two rows, with the anterior eye row slightly procurved, and the posterior eye row slightly recurved (Fig. 5E); eye group 0.56 long, 1.18 wide; ALE-AME 0.14, AME-AME 0.10, PLE-PME 0.04, PME-PME 0.36; MOA 0.43 long, front width 0.43, back width 0.70; ALE: AME: PLE: PME (0.34: 0.19: 0.23: 0.16). Three slightly thick setae on clypeus (Fig. 5E). Chelicerae dark brown (dorsal view); inner margin with three teeth, outer margin with seven teeth. Labium, coxae of palp and sternum brown (Fig. 5C). Labium 0.53 long, 0.80 wide, with four conspicuous cuspules. Coxae of palp 1.50 long, 1.10 wide, with approx. 13 conspicuous cuspules (the right one, ventral view) (Fig. 5C). Sternum 2.97 long, 2.35 wide, with a large smooth area which lacks setae in the center, but many setae outside this area (Fig. 5C).

Legs brown, with long and short brown sparse setae. Basal part of tibia III with saddle-like depression dorsally (Fig. 5F). Palp with a single tarsal claw, with two denticles on the claw. Legs each with three tarsal claws, paired claws with one denticle. Leg formula: IV, I, III, II. Measurements: palp $6.86(2.63+1.25+1.42+1.56)$, leg I 8.11 $(2.97+1.46+1.83+0.99+0.86)$, leg II $7.18(2.55+1.36+1.54+0.84+0.89)$, leg III 7.46 $(2.92+0.92+1.45+0.92+1.25)$, leg IV $8.64(3.07+1.22+1.62+1.42+1.31)$. Leg II and leg III are almost the same length.

Opisthosoma ellipsoid and black, scattered with many slender, short black setae. Spinnerets brown (Fig. 5D), PMS short and one-segmented, 0.48 long, PMS-PMS 0.08; PLS divided into three sections, 0.78 long. Genitalia with a pair of spermathecae, terminating with face-to-face bowl-shaped lobes; stalks sclerotized distally, each stalk sturdy, short, simple and direct, without the trench between the distal part of the stalks and the lobes (Fig. 5G). 
Variation. The female genitalia show considerable intraspecific variations: the spermathecae stalks of the holotype (Fig. 5G) and some paratypes are unbent (Fig. 6A-C, E), or slightly curved (Fig. 6D), or the stalk on the left is tilted to the right by ca. $30^{\circ}$, and the right stalk is curled distally (Fig. 6F). The spermathecae of all samples are face to face, except for one (Fig. 6F).

Male. Unknown.

Etymology. The species epithet, a noun in apposition, refers to the type locality.

Distribution. Hainan Province (Baisha County, Changjiang County, Dongfang City, Wuzhishan City).

GenBank accession numbers. LH-2017-080: MK454955; LH-2017-089: MK454956; LH-2017-090: MK454957; LH-2017-128: MK454958; LH-2017-135: MK454959; LH-2017-136: MK454960; LH-2017-161: MK454961.

Remarks. The mean intraspecific genetic distance of C. baisha sp. n. is $1.25 \%$ and $1.23 \%$ using Kimura 2-parameter (K2P) model and $p$-distance model, respectively. The interspecific genetic distance between $C$. baisha sp. n. and C. baoting sp. n. is $5.78 \%$ and $5.49 \%$ using K2P and $p$-distance, respectively. This interspecific genetic distance in Conothele is comparable to other mygalomorphs identified at 5-6\% (Hamilton et al. 2011, 2014).

\section{Conothele baoting sp. $\mathrm{n}$.}

http://zoobank.org/BB49CB7D-E6A6-4994-A5F3-3F63720DFFA5

Figs 7,8

Holotype. Female (LH-2017-205), collected in Maoding Village, Shiling Town, Baoting County, Hainan Province, China, 18.6987N, 109.7563E, 160 m a.s.l., 20 August 2017, collected by FX Liu, D Li, X Xu (CBEE).

Paratypes. 1 female (LH-2017-209), collected at the same locality as the holotype (CBEE); 5 females (LH-2017-165, LH-2017-166, LH-2017-167, LH-2017-168, LH-2017-169), collected in Wuzhishan City, Hainan Province, China, 18.8147N, 109.5124E, 260-470 m a.s.l., 12 August 2017, collected by FX Liu, D Li, ZT Zhang, X Xu (CBEE); 4 females (LH-2017-179, LH-2017-180, LH-2017-196, LH-2017-198), collected in Qiongzhong County, Hainan Province, China, 18.9899N, 109.6720E, 190-380 m a.s.l., 14-17 August 2017, collected by FX Liu, D Li, X Xu (CBEE); 1 female (LH-2017-187), collected in Wupo Town, Tunchang County, Hainan Province, China, 19.1380N, 110.0625E, 90 m a.s.l., 15 August 2017, collected by FX Liu, D Li, X Xu (CBEE); 3 females (LH-2017-211, LH-2017-212, LH-2017-213), collected in Baoqian Village, Tianya District, Sanya Ciy, Hainan Province, China, 18.3931N, 109.4224E, 90 m a.s.l., 22 August 2017, collected by FX Liu, D Li, X Xu (CBEE).

Diagnosis. Females of $C$. baoting sp. n. can be distinguished from those of other Conothele species by the spermathecae with plate-shaped lobes, each stalk slender, long, distally sclerotized and thickened, and narrowest in the middle. It can be distinguished from C. baisha sp. n. by long stalks each with an obvious trench between the distal part of the stalk and the lobe (Fig. 7G). Moreover, C. baoting sp. n. can be diagnosed from 

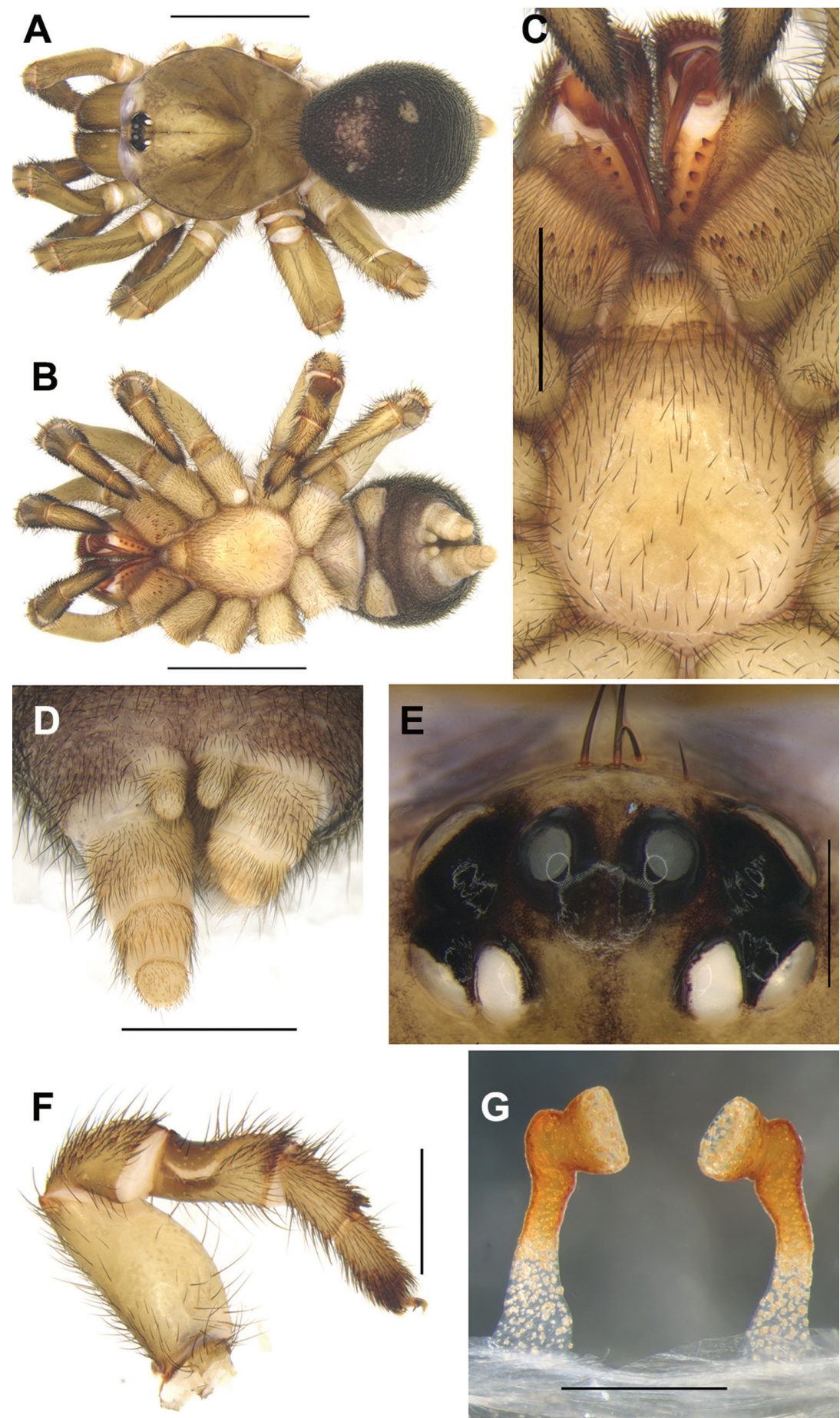

Figure 7. General somatic morphology and female genitalia of Conothele baoting sp. n. holotype (LH2017-205) A dorsal view B ventral view C chelicerae, labium, coxae of palp and sternum, ventral view $\mathbf{D}$ spinnerets, ventral view $\mathbf{E}$ eyes, dorsal view $\mathbf{F}$ left leg III, prolateral view $\mathbf{G}$ female genitalia, dorsal view. Scale bars: $5 \mathrm{~mm}(\mathbf{A}, \mathbf{B}) ; 2 \mathrm{~mm}(\mathbf{C}, \mathbf{D}, \mathbf{F}) ; 0.5 \mathrm{~mm}(\mathbf{E}, \mathbf{G})$. 

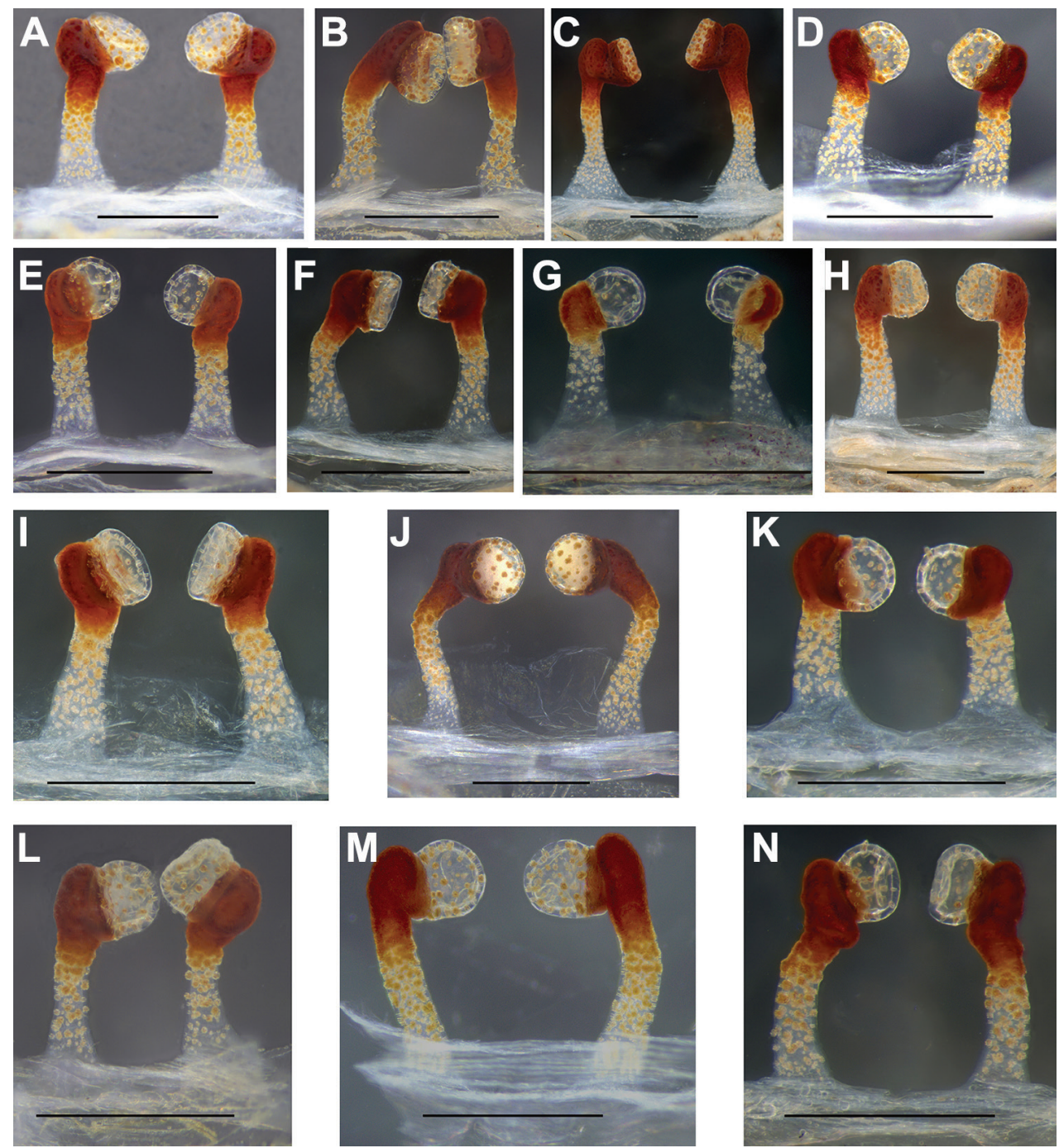

Figure 8. Female genitalia of the paratypes of Conothele baoting sp. n. showing the intraspecific variations in spermathecae A (LH-2017-165) B (LH-2017-166) C (LH-2017-167) D (LH-2017-168) E (LH2017-169) F (LH-2017-179) G (LH-2017-180) H (LH-2017-187) I (LH-2017-196) J (LH-2017-198) K (LH-2017-209) L (LH-2017-211) M (LH-2017-212) N (LH-2017-213) dorsal view. Scale bars: 0.5 mm.

C. baisha sp. n. by the following unique nucleotide substitutions in the standard DNA barcode alignment: G (13), T (97), G (134), A (157), G (172), A (196), T (205), G (223), C (224), T (253), A (280), T (302), A (304), T (322), C (376), G (421), A (424), G (502), A (520), G (592), G (634), A (637).

Description. TL 14.71, chelicerae length 1.80 , carapace 6.92 long, 6.20 wide; opisthosoma 6.52 long, 5.40 wide. Carapace light brown, glabrous, with a few slender setae on or behind the eye tubercle (Fig. 7A). Caput arched. Fovea deep and brown (Fig. 7A). Eye tubercle black. Eight eyes in two rows, with both two eye rows straight (Fig. 7E); 
eye group 0.76 long, 1.39 wide; ALE-AME 0.19, AME-AME 0.25, PLE-PME 0.03, PME-PME 0.57; MOA 0.66 long, front width 0.56, back width 0.90; ALE: AME: PLE: PME (0.38: 0.14: 0.32: 0.15). Three slightly thick setae on clypeus (Fig. 7E). Chelicerae light brown (dorsal view); inner margin with five teeth, outer margin with seven teeth. Labium, coxae of palp and sternum brown (Fig. 7C). Labium 0.84 long, 1.41 wide, with three conspicuous cuspules. Coxae of palp 2.04 long, 1.54 wide, with approx. 18 conspicuous cuspules (the right one, ventral view) (Fig. 7C). Sternum 3.91 long, 3.23 wide, with a large smooth area which has a few setae in the center and many setae outside (Fig. 7C).

Legs brown, with long and short brown dense setae. Basal part of tibia III with saddle-like depression dorsally (Fig. 7F). Palp with a single tarsal claw, with two denticles on the claw. Legs each with three tarsal claws, paired claws with two denticles. Leg formula: IV, I, II, III. Measurements: palp $8.73(3.20+1.58+2.03+1.92)$, leg I 11.15 $(3.99+2.17+2.49+1.49+1.01)$, leg II $10.37(3.58+2.11+2.20+1.22+1.26)$, leg III 9.81 $(3.26+1.41+2.10+1.40+1.64)$, leg IV $11.94(4.13+1.72+2.25+2.17+1.67)$.

Opisthosoma ellipsoid, black, scattered with sparse slender, short black setae. Spinnerets brown (Fig. 7D). PMS one-segmented and short, slightly thick, 0.62 long, PMS-PMS 0.12; PLS divided into three sections, 2.23 long. Genitalia with a pair of spermathecae; spermathecae with plate-shaped lobes, each stalk slender, long, distally sclerotized and thickened, and narrowest in the middle. There is an obvious trench between the distal part of the stalk and lobe (Fig. 7G).

Variation. The female genitalia show considerable intraspecific variations: the stalks of some specimens are unbent (Fig. 8A, D, E, G, H, I, K, L), while others are slightly curved (Fig. 8B, C, F, J, M, N); there are three different shapes of lobes of spermathecae, slightly globular (Fig. 8D, E, G, J, K, M, N), bowl-shaped (Fig. 8A, B, F, H, I, L), and plate-shaped (Figs 7G, 8C).

Male. Unknown.

Etymology. The species epithet, a noun in apposition, refers to the type locality.

Distribution. Hainan Province (Baoting County, Qiongzhong County, Sanya Ciy, Tunchang County, Wuzhishan City).

GenBank accession numbers. LH-2017-165: MK454962; LH-2017-166: MK454963; LH-2017-167: MK454964; LH-2017-168: MK454965; LH-2017-169: MK454966; LH-2017-179: MK454967; LH-2017-180: MK454968; LH-2017-187: MK454969; LH-2017-196: MK454970; LH-2017-198: MK454971; LH-2017-205: MK454972; LH-2017-209: MK454973; LH-2017-211: MK454974; LH-2017-212: MK454975; LH-2017-213: MK454976.

Remarks. The mean intraspecific genetic distance of C. baoting sp. n. is $0.77 \%$ in $\mathrm{K} 2 \mathrm{P}$ and $0.76 \%$ in $p$-distance.

\section{Acknowledgements}

This study was supported by the grants from the National Natural Sciences Foundation of China (NSFC) (31272324; 31601850), the Hunan Provincial Natural Science Foundation of China (2017JJ3202), and the Singapore Ministry of Education AcRF 
Tier 1 grant (R-154-000-A52-114). We thank Fan Li, He Zhang, Xuan Huang, and also the staff of the Centre for Behavioural Ecology and Evolution (CBEE, Hubei University) for all their help and support throughout this study. We also thank Wenjin Gan for her help in the logistics in the field. We value the positive feedback from Chris Hamilton, Vera Opatova, and Rebecca Godwin.

\section{References}

Ayoub NA, Garb JE, Hedin M, Hayashi CY (2007) Utility of the nuclear protein-coding gene, elongation factor-1 gamma (EF-1g), for spider systematics, emphasizing family level relationships of tarantulas and their kin (Araneae: Mygalomorphae). Molecular Phylogenetics and Evolution 42: 394-409. https://doi.org/10.1016/j.ympev.2006.07.018

Bond JE, Coyle FA (1995) Observations on the natural history of an Ummidia trapdoor spider from Costa Rica (Araneae, Ctenizidae). The Journal of Arachnology 23: 157-164.

Bond JE, Hendrixson BE, Hamilton CA, Hedin M (2012) A reconsideration of the classification of the spider infraorder Mygalomorphae (Arachnida: Araneae) based on three nuclear genes and morphology. PLoS ONE 7(6): e38753. https://doi.org/10.1371/journal.pone.0038753

Brower AVZ (2010) Alleviating the taxonomic impediment of DNA barcoding and setting a bad precedent: names for ten species of 'Astraptes fulgerator' (Lepidoptera: Hesperiidae: Eudaminae) with DNA-based diagnoses. Systematics and Biodiversity 8: 485-491. https:// doi.org/10.1080/14772000.2010.534512

Cook LG, Edwards RD, Crisp MD, Hardy NB (2010) Need morphology always be required for new species descriptions? Invertebrate Systematics 24: 322-326. https://doi.org/10.1071/IS10011

Coyle FA (1981) Notes on the behaviour of Ummidia trapdoor spiders (Araneae, Ctenizidae): burrow construction, prey capture, and the functional morphology of the peculiar third tibia. Bulletin of British Arachnological Society 5: 159-165.

Coyle FA (1985) Ballooning behavior of Ummidia spiderlings (Araneae, Ctenizidae). The Journal of Arachnology 13: 137-138.

Decae AE (2010) The genus Ummidia Thorell 1875 in the western Mediterranean, a review (Araneae: Mygalomorphae: Ctenizidae). The Journal of Arachnology 38: 328-340. https:// doi.org/10.1636/A09-85.1

Doleschall L (1859) Tweede Bijdrage tot de kennis der Arachniden van den Indischen Archipel. Acta Societatis Scientiarum Indica-Neerlandica 5: 1-60.

Fisher JR, Fisher DM, Skvarla MJ, Dowling APG (2014) Pre-ballooning in Ummidia Thorell 1875 (Araneae: Ctenizidae) from the Interior Highlands, USA: second account from the region and review of mygalomorph ballooning. The Journal of Arachnology 42: 318-321. https://doi.org/10.1636/B14-43.1

Folmer O, Black M, Hoeh W, Lutz R, Vrijenhoek R (1994) DNA primers for amplification of mitochondrial cytochrome c oxidase subunit I from diverse metazoan invertebrates. Molecular Marine Biology and Biotechnology 3: 294-299.

Gertsch WJ (1979) American spiders. Van Nostrand Reinhold, New York, 274 pp. 
Godwin RL, Opatova V, Garrison NL, Hamilton CA, Bond JE (2018) Phylogeny of a cosmopolitan family of morphologically conserved trapdoor spiders (Mygalomorphae, Ctenizidae) using Anchored Hybrid Enrichment, with a description of the family, Halonoproctidae Pocock 1901. Molecular Phylogenetics and Evolution 126: 303-313. https://doi.org/10.1016/j.ympev.2018.04.008

Hamilton CA, Formanowicz DR, Bond JE (2011) Species delimitation and phylogeography of Aphonopelma hentzi (Araneae, Mygalomorphae, Theraphosidae): cryptic diversity in North American tarantulas. PLoS ONE 6: e26207. https://doi.org/10.1371/journal.pone.0026207

Hamilton CA, Hendrixson BE, Brewer MS, Bond JE (2014) An evaluation of sampling effects on multiple DNA barcoding methods leads to an integrative approach for delimiting species: A case study of the North American tarantula genus Aphonopelma (Araneae, Mygalomorphae, Theraphosidae). Molecular Phylogenetics and Evolution 71: 79-93. https://doi. org/10.1016/j.ympev.2013.11.007

Haupt J (2006) On the taxonomic position of the East Asian species of the genus Ummidia Thorell, 1875 (Araneae: Ctenizidae). In: Deltshev C, Stoev P (Eds) European Arachnology 2005, Acta Zoologica Bulgarica Supplement 1: 77-79.

Hedin M, Bond JE (2006) Molecular phylogenetics of the spider infraorder Mygalomorphae using nuclear rRNA genes (18S and 28S): conflict and agreement with the current system of classification. Molecular Phylogenetics and Evolution 41: 454-471. https://doi. org/10.1016/j.ympev.2006.05.017

Main BY (1985) Further studies on the systematics of ctenizid trapdoor spiders: A review of the Australian genera (Araneae: Mygalomorphae: Ctenizidae). Australian Journal of Zoology (Supplementary Series) 108: 1-84. https://doi.org/10.1071/AJZS108

Opatova V, Bond JE, Arnedo MA (2013) Ancient origins of the Mediterranean trap-door spiders of the family Ctenizidae (Araneae, Mygalomorphae). Molecular Phylogenetics and Evolution 69: 1135-1145. https://doi.org/10.1016/j.ympev.2013.08.002

Ortiz D (2007) Ummidiinae, a new replacement name for Pachylomerinae Simon, 1889 (Araneae: Ctenizidae). Boletín Sociedad Entomológica Aragonesa 40: 395-396.

Planas E, Ribera C (2015) Description of six new species of Loxosceles (Araneae: Sicariidae) endemic to the Canary Islands and the utility of DNA barcoding for their fast and accurate identification. Zoological Journal of the Linnean Society 174: 47-73. https://doi.org/10.1111/zoj.12226

Raven RJ (1985) The spider infraorder Mygalomorphae (Araneae): cladistics and systematics. Bulletin of American Museum of Natural History 182: 1-180.

Siliwal M, Nair MV, Molur S, Raven R (2009) First record of the trapdoor spider genus Conothele (Araneae, Ctenizidae) from India, with a description of two new species. The Journal of Arachnology 37: 1-9. https://doi.org/10.1636/A07-86.1

Tamura K, Peterson D, Peterson N, Stecher G, Nei M, Kumar S (2013) MEGA6: Molecular evolutionary genetics analysis version 6.0. Molecular Biology and Evolution 28: 27312739. https://doi.org/10.1093/molbev/msr121

World Spider Catalog (2019) World spider catalog. Natural History Museum Bern, version 20.0. http://wsc.nmbe.ch [Accessed on 30 January 2019] 
Xu X, Liu FX, Chen J, Li D, Kuntner M (2015) Integrative taxonomy of the primitively segmented spider genus Ganthela (Araneae: Mesothelae: Liphistiidae) - DNA barcoding gap agrees with morphology. Zoological Journal of the Linnean Society 175: 288-306. https://doi.org/10.1111/zoj.12280

Xu X, Xu C, Liu F, Zhang Z, Li D (2017a) Four new species of the trapdoor spider genus Conothele Thorell, 1878 from Mainland China and Laos (Araneae, Ctenizidae). ZooKeys 643: 63-74. https://doi.org/10.3897/zookeys.643.10543

Xu X, Liu F, Ono H, Chen J, Kuntner M, Li D (2017b) Targeted sampling in Ryukyus facilitates species delimitation of the primitively segmented spider genus Ryuthela (Araneae: Mesothelae: Liphistiidae). Zoological Journal of the Linnean Society 181: 867-909. https://doi.org/10.1093/zoolinnean/zlx024

Yang Z, Xu X (2018) Two new species of the trapdoor spider genus Conothele Thorell, 1878 (Mygalomorphae: Halonoproctidae) from China. Zootaxa 4442: 171-180. https://doi.org/10.11646/zootaxa.4442.1.10 